\title{
Methods
}

Michael Karrenbauer*, Stephan Ludwig, Henning Buhr, Henrik Klessig, Anne Bernardy, Huanzhuo Wu, Christoph Pallasch, Amina Fellan, Nicolai Hoffmann, Vasco Seelmann, Maroua Taghouti, Simon Wunderlich, Pedro Tercero Lozano, Andreas Hoell, Christian Stimming, Dhruvin Patel, Savita Seetaraman, Serjoscha Bender, Elena Eberhardt, Thomas Schildknecht, Werner Herfs, Simon Storms, Volker Stich, Norbert Niebert, Hans D. Schotten and Frank H. P. Fitzek

\section{Future industrial networking: from use cases to wireless technologies to a flexible system architecture}

\section{Zukünftige industrielle Vernetzung: Von Anwendungsfällen über Funktechnologien zu einer flexiblen Systemarchitektur}

https://doi.org/10.1515/auto-2018-0141

Received November 23, 2018; accepted February 19, 2019

\begin{abstract}
In this paper, we propose a communication network architecture for industrial applications that combines new $5 \mathrm{G}$ technologies with other existing communication technologies on the shop floor. This architecture connects private and public mobile networks with local networking technologies to achieve a flexible setup addressing many different industrial use cases. We show how the advancements introduced around the new $5 \mathrm{G}$ mobile technology can address a wide range of industrial requirements. We further describe relevant use cases and develop
\end{abstract}

\footnotetext{
*Corresponding author: Michael Karrenbauer, Chair for Wireless Communications and Navigation, University of Kaiserslautern, Kaiserslautern, Germany, e-mail: karrenbauer@eit.uni-kl.de Stephan Ludwig, Henrik Klessig, Robert Bosch GmbH, Renningen, Germany, e-mails: stephan.ludwig2@de.bosch.com, henrik.klessig@de.bosch.com

Henning Buhr, Pedro Tercero Lozano, Dhruvin Patel, Savita Seetaraman, Serjoscha Bender, Norbert Niebert, Ericsson $\mathrm{GmbH}$, Herzogenrath, Germany, e-mails:

henning.buhr@ericsson.com, pedro.tercero.lozano@ericsson.com, dhruvin.patel@ericsson.com, savita.seetaraman@ericsson.com, serjoscha.bender@ericsson.com, norbert.niebert@ericsson.com Anne Bernardy, Vasco Seelmann, Volker Stich, FIR e. V. at RWTH Aachen, Aachen, Germany, e-mails: anne.bernardy@fir.rwth-aachen.de, vasco.seelmann@fir.rwth-aachen.de, volker.stich@fir.rwth-aachen.de Huanzhuo Wu, Maroua Taghouti, Simon Wunderlich, Frank H. P. Fitzek, Deutsche Telekom Chair of Communication Networks, Technische Universität Dresden, Dresden, Germany, e-mails: huanzhuo.wu@tu-dresden.de, maroua.taghouti@tu-dresden.de, simon.wunderlich@tu-dresden.de,frank.fitzek@tu-dresden.de
}

an overall communication system architecture proposal, which is able to fulfill not only technical requirements but also system requirements, which result from specific applications existing in today's and future manufacturing scenarios.

Keywords: $5 \mathrm{G}$ mobile communication, heterogeneous networks, industrial communication, Industrial Internet of Things, machine-to-machine communications, network architecture, network function virtualization, network slicing, software defined networking, Multi-RAT

Zusammenfassung: In diesem Paper wird eine Architektur für Kommunikationsnetze für industrielle Anwendungen vorgestellt, die neue $5 \mathrm{G}$-Technologien mit vorhandener Kommunikationstechnik auf der Feldbusebene kombiniert. Diese Architektur verbindet private und öffentliche Mobilfunknetze mit lokalen Funktechnologien, um einen flexiblen Aufbau zu ermöglichen, der in der Lage ist, viele industrielle Anwendungsfälle zu unterstützen. Es wird gezeigt, wie die Errungenschaften, die mit der neu-

Christoph Pallasch, Nicolai Hoffmann, Werner Herfs, Simon Storms, Laboratory for Machine Tools and Production Engineering, RWTH Aachen, Aachen, Germany, e-mails:

c.pallasch@wzl.rwth-aachen.de,n.hoffmann@wzl.rwth-aachen.de, w.herfs@wzl.rwth-aachen.de,s.storms@wzl.rwth-aachen.de Amina Fellan, Hans D. Schotten, Chair for Wireless Communications and Navigation, University of Kaiserslautern, Kaiserslautern, Germany, e-mails: fellan@eit.uni-kl.de, schotten@eit.uni-kl.de Andreas Hoell, Christian Stimming, SICK AG, Waldkirch, Germany, e-mails: andreas.hoell@sick.de, christian.stimming@sick.de Elena Eberhardt, Thomas Schildknecht, Schildknecht AG, Murr, Germany, e-mails: elena.eberhardt@schildknecht.ag, thomas.schildknecht@schildknecht.ag 
en 5G-Technologie eingeführt werden, einen großen Bereich der industriellen Anforderungen erfüllen können. Weiterhin werden relevante Anwendungsfälle beschrieben und eine Gesamtsystemarchitektur vorgeschlagen, welche nicht nur die technischen, sondern auch die funktionalen Anforderungen, welche von den spezifischen Anwendungen heutiger und zukünftiger Herstellungsprozesse gestellt werden, erfüllen kann.

Schlagwörter: 5G Mobilfunk, Heterogene Netze, Industrielle Kommunikationstechnik, Industrial Internet of Things, Machine-to-machine communications, Netzwerkarchitektur, Network Function Virtualization, Network Slicing, Software Defined Networking, Multi-RAT

\section{Introduction}

The growing digitalization and interconnection of manufacturing processes is leading to a closer gearing of companies, suppliers and customers. Production becomes increasingly flexible and the connectivity of the devices has to become likewise flexible. This is why $5^{\text {th }}$ generation mobile networks (5G) are considered to be key enablers for new use cases towards the vision of Industry 4.0, such as advanced condition monitoring, predictive maintenance and product quality assurance. In this regard, the research initiative " $5 \mathrm{G}$ : Industrial Internet (5G: II)" [1] of the German Federal Ministry of Education and Research addresses the requirements on a $5 \mathrm{G}$ communication network in industrial production. As a part of this initiative, the collaborative 5Gang research project [2] considers different use cases of future industrial production and their requirements on the communication network. The project scope covers not only local production sites but also opportunities arising from adding inter-site connections. 5Gang is a consortium of eight partners from industry and academia who bring in their experience covering technical, business and production process aspects.

In this paper, we describe a way towards a flexible architecture for industrial networking, which is able to support the heterogeneous networking technologies in today's production facilities while benefiting from $5 \mathrm{G}$ technology at the same time. In Section 2, we summarize related work on $5 \mathrm{G}$ and establish relations to existing architectures. In Section 3, we present use cases along with their technical requirements on $5 \mathrm{G}$ systems, as well as system requirements together with exemplary uses cases, which we use to verify the developed system architecture. We detail our 5Gang architecture, its system components, functions and interfaces in Section 4 and describe in Section 5 , how the system architecture integrates into existing reference architectures. Finally, we draw conclusions in Section 6.

\section{Related work}

The field of industrial information technology and industrial communication already plays an important role in today's production facilities and is increasingly becoming a focus of interest from both academia and industry along with the course of the German "Industry 4.0" initiative. As we will show in the following sections, the fulfillment of requirements of manufacturing use cases necessitates the use of $5 \mathrm{G}$ systems, as all three main capabilities of $5 \mathrm{G}$ are addressed, either by the sheer number of participants in the network (massive Machine Type Communications (mMTC)), by demanding real-time capabilities (UltraReliable and Low Latency Communications (URLLC)) or by demanding very high bandwidth (Enhanced Mobile Broadband (eMBB)).

There are already descriptions of $5 \mathrm{G}$ system architectures, partly covering manufacturing scenarios, which we summarize in the following. Also, industrial use cases and related requirements have been discussed in the literature already. We will provide a short overview in the following.

\subsection{Benefits of $5 \mathrm{G}$}

The standardization of $5 \mathrm{G}$ is planned to be finished by 2020. Its first release, Release 15 , was already approved by 3GPP in June 2018 and first commercial, pre-standard systems were deployed already in 2018. By the end of 2019, 3GPP Release 16 will be frozen. Although requirements might change under way, the overall targets are taking shape and can be considered as a basis for 5Gang. The United Nations organization ITU-R addresses three main capabilities that define the requirements for $5 \mathrm{G}$ [3]:

1. eMBB will provide up to $20 \mathrm{Gbit} / \mathrm{s}$ of data towards the end-users (100 Mbit/s per user) and $10 \mathrm{Gbit} / \mathrm{s}$ (50 Mbit/s per user) from the end-users towards the network. The user plane latency shall be below $4 \mathrm{~ms}$.

2. URLLC, or critical Machine Type Communications (cMTC), requires a user plane latency of less than $1 \mathrm{~ms}$.

3. mMTC will allow up to $1,000,000$ connected devices per $\mathrm{km}^{2}$ with given Quality of Service (QoS) $[4,5,6]$.

To some extent, existing wireless technologies can fulfill the eMBB and mMTC requirements already today. However, they cannot reliably support the URLLC case. Apart 
from the New Radio (NR) interface [7], which mainly tackles the technical requirements above from a radio design perspective, $5 \mathrm{G}$ will impose massive changes to networking in general and to the core network in particular, too:

- Softwarization and virtualization: The same physical resources (communication, computing, storage) can be used for many use cases. A distributed cloud (DC) allows to move certain nodes of the mobile network closer to the end-user, which, in the case of a production environment, can even mean core network nodes. From an economic standpoint, this would not be reasonable with high-performing, specialized hardware. Here, the virtualization techniques reduce costs: Instead of installing expensive hardware in the factory, 3GPP has standardized the option to execute certain virtualized user plane functions inside the factory. This allows to keep data inside the factory, which is an important privacy aspect, and reduces network latency [8].

- Software Defined Networking (SDN): SDN [9] enables network elements to be controlled by a central intelligent management platform, enabling dynamic and flexible information routing. Based on SDN, network slicing $[10,11]$ dynamically reserves resources per application and provides the required QoS, improving system reliability and flexibility (see, e. g., [12] for the condition monitoring use case).

- Mesh network: In a mesh network [13], each node can have a direct connection to several neighbor nodes and cooperate with them to efficiently route data. Mesh networks are self-organizing, self-healing and provide a high robustness against link/node loss. Also, the communication range and resilience can be increased while enabling efficient device-to-device communication.

Other advantages of $5 \mathrm{G}$ for industrial networking are:

- Mobility support: Moving workpiece carriers can be controlled or tracked not only inside the factory but also on their way between different production sites.

- Energy efficiency: 5G allows operating times of 10 years for transmitting small volumes of data from battery-powered devices in an energy-efficient manner.

- Security: SIM cards (or alternatives) provide a secure way to manage devices and restrict network access.

- Economy of scale: The large 5G ecosystem will increase the volume of the radio modems leading to cheaper equipment, which would not be possible if every production solution used separately specialized hardware.

\section{$2.25 \mathrm{G}$ architecture descriptions}

In the following, we provide an overview of the $5 \mathrm{G}$ architecture descriptions existing today and will investigate, how our proposed architecture aligns with them.

\subsubsection{NGMN 5G architecture for verticals}

The " $5 \mathrm{G}$ White Paper" [14] of the NGMN consortium is one of the first that brought up $5 \mathrm{G}$ for verticals. It addresses verticals and envisions the use cases of massive sensor networks in the Internet of Things (IoT), which is termed "massive IoT", of ultra-reliable communication, and of extreme real-time communication, e. g., for collaborative robots. All three use cases are also considered in the 5Gang project and require security, identity and privacy; real-time, seamless and personalized experience; responsive interaction and charging, as well as QoS and contextual behavior of the system. Beyond connectivity, $5 \mathrm{G}$ may offer services like transparency of connectivity, location, resilience, reliability, and high availability. We design our architecture, such that it integrates with and details the (virtualized) infrastructure resource layer and parts of the business enablement layer of the " $5 \mathrm{G}$ architecture" in $[14$, sec. 5.4].

\subsubsection{GPPP 5G architecture}

A $5 \mathrm{G}$ architecture has been developed by the EU-funded project "5GPPP" in [15]. It primarily focuses on network slicing and the radio access. It also gives a detailed overview of the capabilities of each component and describes the infrastructure, into which the 5Gang architecture well integrates for the use in the factory of the future. The 5GPPP architecture is divided into resource/functional, network and service levels. The resource/functional level provides the physical resources for communication, computation and storage. In addition to the wireless and fixed access it consists of edge cloud and core/central cloud resources. Virtualization of the physical network is achieved by a network operating system and programmable network control units, such that network slices can be built on top. At the service levels, these slices are orchestrated in an end-to-end fashion using management functions of all levels. As our architecture enables network slicing by providing the physical network infrastructure and corresponding management and orchestration interfaces, it integrates well into the 5GPPP architecture. 


\subsubsection{GPP 5G architecture}

3GPP also proposed a first architecture of its $5 \mathrm{G}$ system in [16]. It details the $5 \mathrm{G}$ components on the network side, e. g., base stations and the core network, assuming that 5G-enabled devices will have an anyhow natured 5G modem. In this paper, we embed the architecture of [16] into the factory of the future and abstract it as the "cellular backend".

\subsubsection{G-ACIA 5G building blocks}

The 5G Alliance for Connected Industries and Automation (5G-ACIA) [17] is a global initiative that brings together Operational Technology (OT) industry, Information and Communication Technology (ICT) industry, and academia to provide a common platform for discussing technical, regulatory and business aspects of $5 \mathrm{G}$ for the industrial domain. In their white paper [18], they provide an overview of 5G's basic potential for manufacturing, outline use cases and requirements, and name important $5 \mathrm{G}$ building blocks. There exists a plethora of operational/functional requirements that go far beyond the technical requirements of industrial use cases. In addition to availability and reliability, the networks must feature maintainability, safety and integrity. For example, functional safety considers safety measures that prevent harming people or the environment and which must be integrated as native network services. Towards the success of $5 \mathrm{G}$ in the industrial domain, the 5G-ACIA promotes in [18] the integration of $5 \mathrm{G}$-enabled industrial components with legacy communication technology, the operation of private $5 \mathrm{G}$ networks, seamless handovers between public and private $5 \mathrm{G}$ networks, and end-to-end network slicing across technologies, countries and network operators. 5GACIA particularly names wireless connectivity, edge computing, and network slicing as technologies that make $5 \mathrm{G}$ disruptive for the manufacturing industry. In this paper, we tackle many of the mentioned technologies and integrate them into our proposed architecture for industrial networking.

\subsection{Requirements of industrial use cases}

In the following, we briefly review existing technical requirements derived from industrial use cases on proprietary wireless communications and $5 \mathrm{G}$ systems.

\subsubsection{Requirements derived within the ZDKI programme}

Requirements of industrial use cases have been investigated in research projects of the German funding programme "Reliable wireless communication in industry" (German acronym: ZDKI). For example, in the "HiFlecs" project, requirements of different industrial applications have been grouped into three requirement profiles [19]. In [20], two specific use cases of the "KoI" project and their quantitative requirements have been provided. The results in the "ZDKI" programme have been collected by the accompanying research project (German acronym: BZKI) in the technical group 1: "Applications, Requirements and Validation" and have been published in the report [21]. A thorough compilation of different industrial applications and quantitative requirements has been provided in [22]. The authors showed that the spectrum of applications in an industrial environment is very broad and consequently their requirements are very distinct. The authors conclude that in order to have a suitable system design, different technologies have to be combined purposefully with regard to the specific applications, which we attempt to realize in our proposed architecture, as well.

\subsubsection{Requirements from 3GPP TR 22.804}

In preparation of Release 16, 3GPP performed a study on communication for automation in Vertical Domains. The resulting report [23] names use cases and their requirements for the 3GPP $5 \mathrm{G}$ system. One major part is on the use case class "factories of the future" in general and on factory automation in particular, as well as on industrial security requirements. Within these sections, the report [23] addresses similar use cases as $5 \mathrm{Gang}$, and the technical requirements of 5Gang and [23] are well-aligned such that this paper can be considered to detail the first approach of the principle architecture in [23].

\section{Use cases and requirements}

In the following, we review some exemplary use cases and technical requirements of $5 \mathrm{G}$ which have been developed by $3 \mathrm{GPP}$ and within our $5 \mathrm{Gang}$ project. All use case classes of [23], except for the "process automation" class, are considered and fulfilled when designing the overall network architecture. However, for brevity, we only introduce the ones, for which we exemplary will check that their system requirements are fulfilled by the proposed system ar- 
chitecture (cf. Section 4). Whereas the focus of 3GPP is on the use cases' technical requirements, in Section 3.2 we derive and highlight functional system requirements, which are important for the design of our system architecture.

\subsection{Example use cases and technical requirements}

We now describe some use case classes of 3GPP and give a more application-specific view compared to [23]. We only touch technical requirements of our use cases by highlighting some values and classifying them into the eMBB, URLLC and MMTC categories (see Section 2.1). Safety requirements are mostly omitted for the sake of brevity, as a detailed safety architecture is left as future work. At first, $5 \mathrm{G}$ can be used to extend the field of application in an innovative way while ensuring backward compatibility. A second use case class focuses on mobile robots and mobile material supply in a factory for mounting or distribution. The third use case class focuses on the production operations themselves. Applications like tracking and tracing systems, as well as flexible production without any wired connected machines are examples within this class. The fourth class addresses the control of the processes of the production and logistics.

\subsubsection{Use case class 1: Infrastructure retrofit}

The benefits of $5 \mathrm{G}$ for infrastructure retrofits are included in the use case "connectivity on the factory floor" of [23]. Within 5Gang we divide this use case into three subproblems: First, existing sensor and actor technologies can be enhanced by their integration with mobile communication technology modules. In this regard, $5 \mathrm{G}$ is planned to be more energy-efficient than, e. g., LTE and provides a wider coverage than Bluetooth Low Energy and the-like. This use case class contains all kinds of combinations of sensor stacks (AUTO-IDs, cameras, condition sensors, etc.) with $5 \mathrm{G}$ modules. Here, the potential for large-scale infrastructure retrofits and improved, wireless connectivity between retrofitted sensors and actuators is unlocked by both, 5G's high bandwidth (eMBB) and ability for critical communication (cMTC or URLLC). Second, existing communication infrastructure is often very weak in many terms, e.g., in old production facilities without a proper IT infrastructure or in large production areas without Internet connection in the countryside. Here, $5 \mathrm{G}$ provides a more reliable network than LTE and can transfer critical data. The third case addresses information multiplexing, e. g., in a machine. The retrofit of communication systems in machines by $5 \mathrm{G}$ allows outsourcing of the multiplexing process by network slicing, so that configurations of the machine can be performed independently from the machine and its use case.

Within 5Gang, we extended this use case (class) by the process automation-related use case "decentralized measurement network", e. g., devices, which measure the gas flow through pipelines in remote areas for central gas flow monitoring and regulation. Due to long distances between measurement spots, e. g., across countries, and decentralized equipment, $5 \mathrm{G}$ can provide connectivity to the sensors. In areas of no coverage, few sensors can act as $5 \mathrm{G}$ gateways, where the sensors are connected with each other using another mesh network technology. Since the pipeline infrastructure already exists, we classify it into retrofit. Here, a latency of the information multiplexing processes is essential, such that the $1 \mathrm{~ms}$ provided by $5 \mathrm{G}$ is the key potential for this use case (URLLC).

\subsubsection{Use case class 2: Mobile robots}

This class includes applications with different automated driven vehicles, like automated guided vehicles (AGVs), which transport material in a dynamic environment. In a well-planned production, the material flow follows a milk run principle with dynamic changes of the milk run route. In some cases, AGVs can be controlled in a master-slave relation, in which only the master AGV communicates with the production system and the slave-AGVs are connected to the master through their own network.

The most challenging scenario is "Distributed Indoor SLAM” (SLAM: Simultaneous Location And Mapping), in which AGVs or mobile production robots reliably navigate in a spatially varying production environment using highprecision navigation based on real-time maps, as shown in Fig. 1. Each AGV provides its own measurements for other devices to create the common map. The mapping needs to be timely synchronized (within less than $50 \mathrm{~ms}$ of latency) and shared among the devices, so that they can simultaneously locate themselves and plan further movements. The necessity of using $5 \mathrm{G}$ technology arises from the real-time requirement of the served application (URLLC). Furthermore, high bandwidth requirements exist (eMBB). Also, computing power, e. g., using a local (edge) cloud is needed for generating the timely synchronized maps in real-time. 


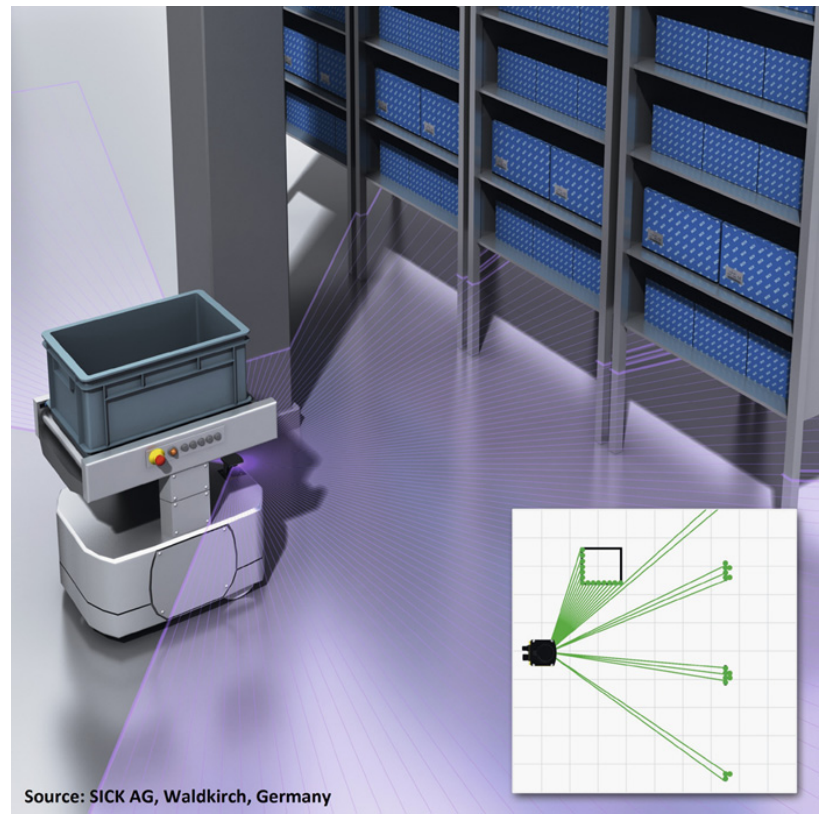

Figure 1: Distributed Indoor SLAM.

\subsubsection{Use case class 3: Inbound logistics for manufacturing, flexible and modular assembly area, plug-and-produce}

Due to the transition to flexible manufacturing and customization of the production processes, companies increasingly ask for a dynamic order-change request system. This requirement demands reliable tracking of the desired product during the entire supply chain process. Hence, "Track and Trace" applications cover orders and material flow across different locations of the supply chain and include sub-use cases such as the following: A companyindependent tracking via an Auto-ID combined with mobile communication technologies like 5G, as well as dynamic allocations for material in different production environments (different facilities). Attaching sensors to workpiece carriers provides a solution approach to track and trace each workpiece carrier independently when connected via a mobile network. This redesigned sensor-stack using $5 \mathrm{G}$ can be realized independently for each workpiece carrier across company locations, even on global logistic routings. As a result, the customer will have an overview of the exact location of his product and which modifications can still be ordered.

The second problem that requires a shared tracking and tracing system is the stocking of material in a shared warehouse. So far, the delivery of materials requires a manual check-in followed by the search for a suitable stocking place in a warehouse. To optimize this, a shared, digitalized, 5G-connected warehouse provides a solution approach.

In 5Gang another use case has been identified, which tracks the product quality along the production line. Additional processing of defective parts is costly and should be avoided. So, the use case aims to detect defects in parts at earlier stages through inline quality control. Corrective measures can be taken and necessary post-production can be initiated before final inspection is completed. A similar case occurs when tracking items within a logistics chain. Here, the transport conditions (e.g., cooling temperature) are monitored continuously. In the event of significant deviations, a re-order can be made before the items arrive. In the former example, tracking of items is performed within a production facility, whereas in the latter, the item is tracked around the world. In both example, sensors, placed on or near the item, on the workpiece or on the workpiece carrier, transmit their measured values (temperature, humidity, shock, vibrations) to a central point for evaluation. The benefit of using $5 \mathrm{G}$ technology arises from the high amount of participants in the network (mMTC).

\subsubsection{Use case class 4: Massive wireless sensor network and process monitoring}

In production, unexpected machine defects cause downtimes, which result in high costs and delays in delivery, and the quality of products depends on the condition of machines which produce them. Automated supervision of machine conditions can prevent unplanned defects, enable planning of maintenance activities, and support continuous product quality control. Distributed sensing of condition values, e. g., noise recordings, offers a solution for anomaly detection. Collecting such data from several locally uncorrelated sources requires a large number of sensors. The $5 \mathrm{G} \mathrm{mMTC}$ profile is optimized towards these requirements in order to build an Industrial Internet of Things (IIoT) network by supporting up to 1,000,000 devices per $\mathrm{km}^{2}$, and dedicated frequency bands allow for independence from the existing infrastructure. This use case requires high mobile broadband connectivity because sensor technologies like high resolution cameras need to be integrated into the machines. If the production process contains many different machines with various connected sensors and actors, the number of connected devices rises and a transmission technology like $5 \mathrm{G}$ is necessary to ensure a reliable IIoT. In order to realize services, such as predictive maintenance, condition monitoring or anomaly detection, sensor nodes need to be allocated to 
dynamic processes and to be mobile over different locations.

In a specific use case, audio data recorded by microphones shall be sent to a central evaluation unit (e. g., in a cloud), which detects anomalies in this data. The sensors have limited mobility and can be distributed across the entire production hall. They can change their positions occasionally (e.g., during reconfigurations or rebuilds) and they are required to have a long battery life, since they can be placed at locations that are difficult to access. The massive amount of wireless sensor nodes will require the $5 \mathrm{G}$ mMTC profile as an enabler. Furthermore, a proper device management must be installed to manage and update the wireless sensor participants and to control access rights.

\subsection{Functional system requirements}

The functional requirements on the system basically equal the main reasons for a plant manager to introduce the new network system. In principle, the new system must have a good overall return on invest within only a few years and shall be future-proof. The return typically comes either from higher production quantity at the same fixed effort (increased efficiency), from a higher degree of automation or from slimmed processes, which in turn increase production quantity, too. As we do not see the one and only killer use case, which will pay off the invest, the system needs to accumulate benefits from different use casesand the collection of beneficial use cases will for sure differ from plant to plant. The invest includes effort for purchasing and operating the network, for retrofitting solutions, and necessary adoptions of existing systems and processes. A smooth transition from legacy solutions to new technology, e. g., 5G, is necessary, requiring interoperability across systems and technologies. Salient requirements are the following:

Flexible and Interoperable Infrastructure: The system shall support all use cases with one common and interoperable infrastructure, which can be deployed worldwide. User equipment surely will implement only subsets of the standard, which are appropriate for the specific application, as not all technical requirements of all use cases can be fulfilled at a time, e. g., high data rate with long battery lifetime.

Flexible Network Ownership and Operation: This means in particular:

1. Easy and fast deployment, operation and management in small and medium-size companies, which do not want to afford owning the network infrastructure and which want to use an operator instead, as well as in large-size companies, which might want to operate their own network infrastructure.

2. Private, sensitive production data (the key value and know-how of production) shall stay on-premise and private. Data shall be shared only through welldefined channels, e. g., across different plants.

3. Thus, private networks shall be supported in licensed (local/regional licensed spectrum or sub-licensed from MNO) and in unlicensed bands ( $2.45 \mathrm{GHz}$ WLAN).

4. In case the manufacturer does not setup a private network, public infrastructure shall provide a local breakout without the operator being able to access the data.

5. System and network operation/management with minimum human/operator intervention and automatic interference alignment between neighboring plants (public-private, private-private).

\section{Functional Requirements derived from the Exemplary}

Use Cases: All use cases have in common:

1. $5 \mathrm{G}$ gateways and sensor nodes shall be easy to install and maintain as costs scale with installation effort.

2. Existing production/process infrastructure shall be securely connected through their existing interfaces (fieldbus, Industrial Ethernet, etc.) and $5 \mathrm{G}$ modems in order to become a part of the overall network.

3. The infrastructure shall allow to build isolated subnetworks with well-defined transfer paths.

4. The system shall integrate existing infrastructure without adjustments or additional hardware/software for incremental retrofitting. This particularly includes the integration of different wired or wireless access technologies like (Industrial and classic) Ethernet, field bus networks, WLAN, RFID and Bluetooth.

5. The system shall support indoor and outdoor operation with handover. The typical size of an indoor shop floor is some $100 \mathrm{~m}$ in square.

From use case class 1 ("infrastructure retrofit"):

1. All infrastructure shall be wirelessly covered. If some area is not covered by a base station, device-to-device or mesh network technologies shall be supported.

2. Machines and devices shall be managed remotely, e. g., by a cloud service.

From use case class 2 ("inbound logistics"):

1. The overall network shall support intra-site connections and (seamless) connectivity along the logistics routes.

2. Localization shall be supported with required accuracy ranging from $1 \mathrm{~m}$ to $1 \mathrm{~cm}$ in three dimensions. 
From use case class 4 ("massive wireless sensor networks"):

1. The system shall manage non-cellular radio subsystems, such that intra-system interference is avoided.

2. In order to extend coverage and to handle high numbers of devices, gateways shall support mesh network technologies or $5 \mathrm{G}$ device-to-device.

3. The system shall support user equipment to establish multiple connections to different base stations in order to balance data traffic and to increase reliability.

\section{5Gang system architecture}

The proposed architecture and its involved components, which are depicted in Fig. 5, have been designed to meet the requirements derived above while assuming 3GPP's 5G will fulfill all their technical requirements in each flavor, eMBB, URLLC and mMTC. In the following, we present the architecture along with descriptions of its components.

\subsection{Overview}

Apart from the improvements and specifications mentioned in Section 2, our architecture covers not only the $5 \mathrm{G}$ context but also technologies already in use in today's manufacturing facilities. This flexible combination of technologies to enable specific use cases forms, to the best of the authors' knowledge, a unique characteristic. The network system is a system of systems, where typically functions are already mapped onto components. Hence, and for the ease of description, we combine components, the functions they provide and the respective interfaces into one description. As has been described in Section 2, softwarization and virtualization will make the alignment of component and function arbitrary in many cases, which backs the reasons.

\subsection{G RAN and core network}

On a very high level the network consists of a core and a radio network. $5 \mathrm{G}$ will provide a new architecture [24] building on a completely new core network, $5 \mathrm{G}$ Core, and an optimized radio network, 5G RAN. Normally the network is operated by Mobile Network Operators (MNO) because they own the frequency spectrum. If a factory owns or leases the spectrum it can as well operate an own network. There is also the option to run only critical compo- nents as private infrastructure on-premise and connect it to a public network, which is managed by a MNO. In the scope of this paper we assume that the network supports public/private operations with all the respective requirements given in Section 3.2, as well as converge with other network technologies like WLAN, fieldbus and Industrial Ethernet by the use of the proposed Aggregation Point.

The $5 \mathrm{G}$ RAN is often called $5 \mathrm{G}$ New Radio ( $5 \mathrm{G}$ NR). It uses an optimized radio interface with a much higher bandwidth compared to the 4G RAN. For sensors with a very low bandwidth it can as well operate in a more energy efficient setup. For more stringent URLLC use cases, sensors and actuators are most likely be connected via a $5 G$ NR interface or a wired network.

The $5 \mathrm{G}$ Core network will be built on cloud native software meaning among other advantages that the SW is better adapted for virtualization. This will enable the implementation of smaller software components for smaller factories in less powerful, smaller data centers.

\subsection{Aggregation point (AP)}

The aggregation point (AP) is the core of our proposed architecture. It is primarily responsible for providing IPbased connectivity of the networks with large numbers of sensors, controllers, and actuators, all of which could be using different wireless/wired technologies, to the core $5 \mathrm{G}$ network and/or to the cloud. The AP is thus to be located at the edge of the network and includes different wireless, as well as wired interfaces. The AP employs SDN solutions to manage the traffic within and between its connected networks. It comprises of an SDN-capable switch (e. g., Open vSwitch [25]) to facilitate the traffic management, and an SDN controller (e. g., Ryu, OpenDaylight) to obtain a centralized logical view of the network. A radio management system entity is also part of the AP. It manages a large number of connected wireless nodes while implementing selforganization network techniques to optimize the overall network performance. Regarding the functional system requirements derived in Section 3.2, the aggregation point is able to address the following demands:

- The AP provides a gateway functionality, i.e., it allows the integration of heterogenous radio technologies while maintaining transparent routing.

- It provides an interface between the fieldbus domain and the network domain, while considering the different network properties.

- It offers different wireless interfaces, thus providing 5G-capability to existing hardware, i. e., in a brownfield scenario. 
- Being equipped with several wireless interfaces, the AP allows to provide seamlessness during handover procedures and to improve reliability and/or data rate by exploiting interface diversity.

\subsection{IloT edge gateway}

The IIoT edge gateway primarily provides connectivity to networks with a large number of sensors, controllers and actuators, all of which can use different wireless/wired technologies. It is thus located at the edge of the $5 \mathrm{G}$ network close to the data provider and at the edge of the network leading from the machine to the cloud. The main task of the gateway is to collect, edit and reduce the data available (at the edge), thereby optimizing the transport by the network in terms of speed and costs, which distinguishes it from the transparent routing in the aggregation point. It interfaces sensors and actuators and provides them with further connectivity, e. g., to a (edge) cloud service or a service within the production environment/company network. Therefore, it contains a $5 \mathrm{G}$ modem and includes different wireless as well as wired interfaces like analog/digital I/Os (e. g., classic 4 to $20 \mathrm{~mA} / 0$ to $10 \mathrm{~V}$ ), (Industrial) Ethernet and Ethernet-based fieldbuses (PROFIBUS, Sercos III, CAN, Modbus, etc.) or wireless (Bluetooth low energy, IEEE $802.11 \mathrm{WiFi}$ ). As a special case, a $5 \mathrm{G}$ device-to-device link is used, where sensors and actuators share the $5 \mathrm{G}$ connection of the gateway. Furthermore, the gateway contains storage and processing resources, which might offer edge cloud services to the connected devices. From a production point of view, the AP shall interface to the process, i.e., collect the operating and diagnostic data of the machine or its sensors together with a time stamp. The compute and storage resources provide maximum flexibility to the user: The data can be cached, pre-processed and analyzed on-site such that, e. g., only alarms are forwarded through the $5 \mathrm{G}$ interface. In addition, the type of transmitted data and the size of packets can be dynamically managed and different modes of link operation can be used in order to adjust connectivity costs: an online mode with permanent connection; an interval mode which transmits only regularly at specified periods; and a sleep mode where the device transmits only if needed. Furthermore, classic IT security mechanisms can be implemented using transport layer and end-to-end encryption. As special cases, the processed data can be sent through the mobile radio provider with the strongest signal ("unsteered roaming"). The attached devices are typically managed via a cloud service (device cloud). An extremely stripped-down version of the IIoT edge gateway could be wireless sensor/actor devices themselves, which build up a mesh network among each other.

\subsection{Device cloud}

The device cloud handles tasks such as user, device and access rights management and sends new settings to the device. Furthermore, it lets users further process data using a standardized API (e. g., RESTful), in an Enterprise Resource Planning (ERP) system or with data cloud services. Further functions are alerting, sending and receiving confirmation via SMS, field strength display of the current data connection, and creation of reports and their delivery to authorized recipients.

\subsection{Non-cellular radio management}

The radio management entity gathers context information about the radio environment and particularly about the link quality of the wireless links associated with the AP. This information can be used to apply self-organization mechanisms to the overall connectivity solution using the SDN capabilities of the AP in order to achieve a complication-free operation of different wireless technologies. With this, the functional requirement of providing interference alignment, derived in Section 3.2, is fulfilled. Moreover, utilizing several wireless interfaces, interface diversity can be exploited, which in turn leads to an improved reliability and/or to an increased data rate, which are functional requirements of many industrial use cases.

One example is shown in Fig. 2. A device, in this case a controller, is connected to the AP using two independent WLAN adapters. In a first step, only one wireless link is established using one WLAN interface, which occupies channel A. The SDN switch is configured in a way, such that traffic going through this interface is routed towards the cloud. The second WLAN interface is used for wireless monitoring. Both interfaces send context information about the current wireless status to the radio resource management. Using this information, if the utilization of a different channel would be beneficial, a second WLAN link is established using channel B. The SDN switch is reconfigured, so that the second wireless interface is now routed to the cloud. After this, the first wireless link can be shut down. This process is "make before break" and works seamlessly, i. e., without packet loss. It is also possible to improve this approach with multipath techniques such as Multipath TCP or parallel redundancy protocol (PRP). The intelligent Radio Management entity also enables to align interference between private and public networks, and be- 


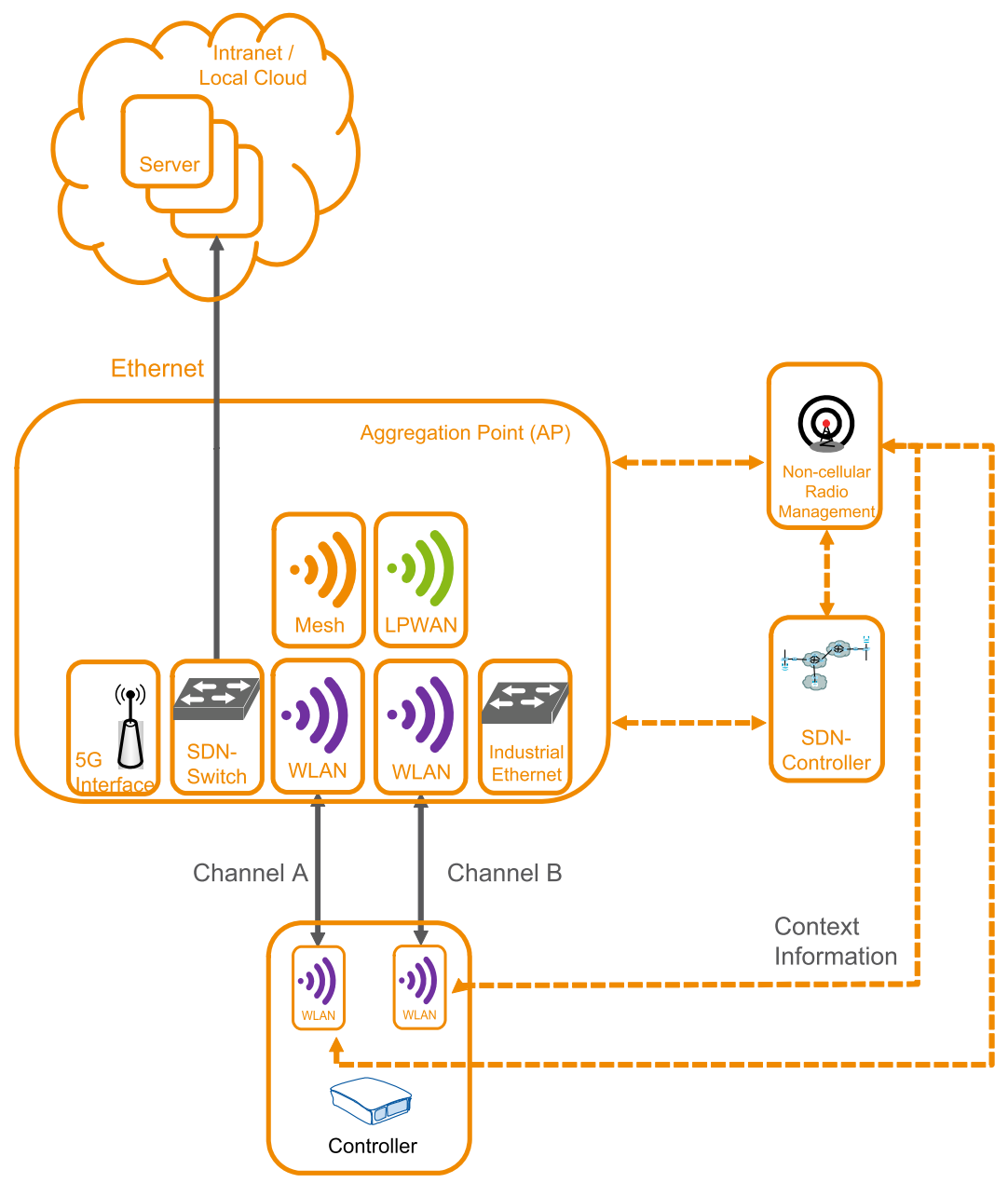

Figure 2: Example for using the "Radio Management" Entity.

tween private networks, which increases the robustness of the communication and reduces human and operator intervention. The latter itself reduces costs for the plant owner.

\subsection{SDN controller}

The SDN controller is the centralized management unit of the network. It acts as a strategic controller in the SDN network, manages the flows inside this network and computes the route which a flow has to follow. In order to do so, it manages the flow-tables on SDN switches which are along this route. With the help of an SDN controller, it is possible to configure the network dynamically so that it meets the changing needs related to configuration, security and optimization. The most popular protocols used by SDN controllers to communicate with SDN switches are OpenFlow and Open vSwitch data-base (OVSDB). OpenDaylight and Ryu are two typical SDN controllers.

\subsection{SDN switch}

An SDN switch is a device, which receives, sends and forwards data packets in a network in order to meet specific requirements. It follows the rules in a flow-table, which is managed by the SDN controller via SDN protocols, like OpenFlow. There are some virtual SDN switches, e. g., Open vSwitch (OVS), to provide a switching stack for hardware virtualization environments. In case high switching capacity is needed, dedicated SDN hardware switches are available which are offering enhanced SDN performance.

\subsection{Tracing of requirements}

With the exception of localization, all functional system requirements of all use cases, and especially those of the exemplary ones shown in this paper, are fulfilled by the communication system architecture. Table 1 shows a summary of the requirements trace. 
Table 1: Functional system requirements and fulfilling component.

\begin{tabular}{ll}
\hline Functional system requirements & Fulfilled by component \\
\hline Integration of heterogeneous radio technologies, transparent routing & Aggregation Point \\
Interface between “fieldbus domain” and “network domain” & Aggregation Point \\
Retrofitting, Support of brown-field scenarios & Aggregation Point, lloT Edge Gateway \\
Separation of private and public data transmissions & SDN Controller, SDN Switches, Private 5G RAN, Private 5G Core \\
Interference alignment, preventing intra-system interference & Non-cellular Radio Management \\
Seamlessness (Handover, Reliability) & Aggregation Point (Multipathing), SDN (Network topology) \\
Very high data rates (uncompressed video streams) & Aggregation Point (Multipathing) \\
IT-Security & Inherent cellular security mechanisms (SIM cards) \\
Remote Management of devices & Device Cloud \\
\hline
\end{tabular}

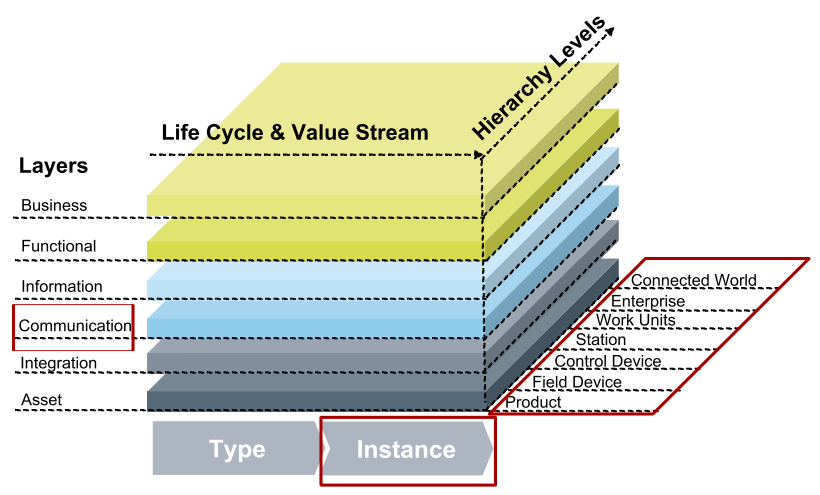

Figure 3: RAMI 4.0 reference architecture.

\section{RAMI 4.0 and IIRA integration}

In the literature, there are similar reference architectures for productions already described and it seems sensible to compare them to the architecture proposed in this paper. For the vertical integration of production technologies, the two most-cited reference architectures for the Industrial IoT (IIoT) [26] are the Industrial Internet Reference Architecture (IIRA) of the Industrial Internet Consortium (IIC) and the RAMI 4.0 architecture of the German "Plattform Industrie 4.0, for which we show ways of integration of our proposed architecture below.

\subsection{RAMI 4.0}

The "Reference Architectural Model Industrie 4.0" (RAMI 4.0) $[27,28]$ has been designed as a future reference model for industrial production and automation to categorize and differentiate different architectural views that are related to each other. The RAMI4.0 (cf. Fig. 3) is structured as a three dimensional model comprised of the axes Hierarchy Levels, Layers and Life Cycle \& Value Stream. The Hierarchy Levels represent the classical automation

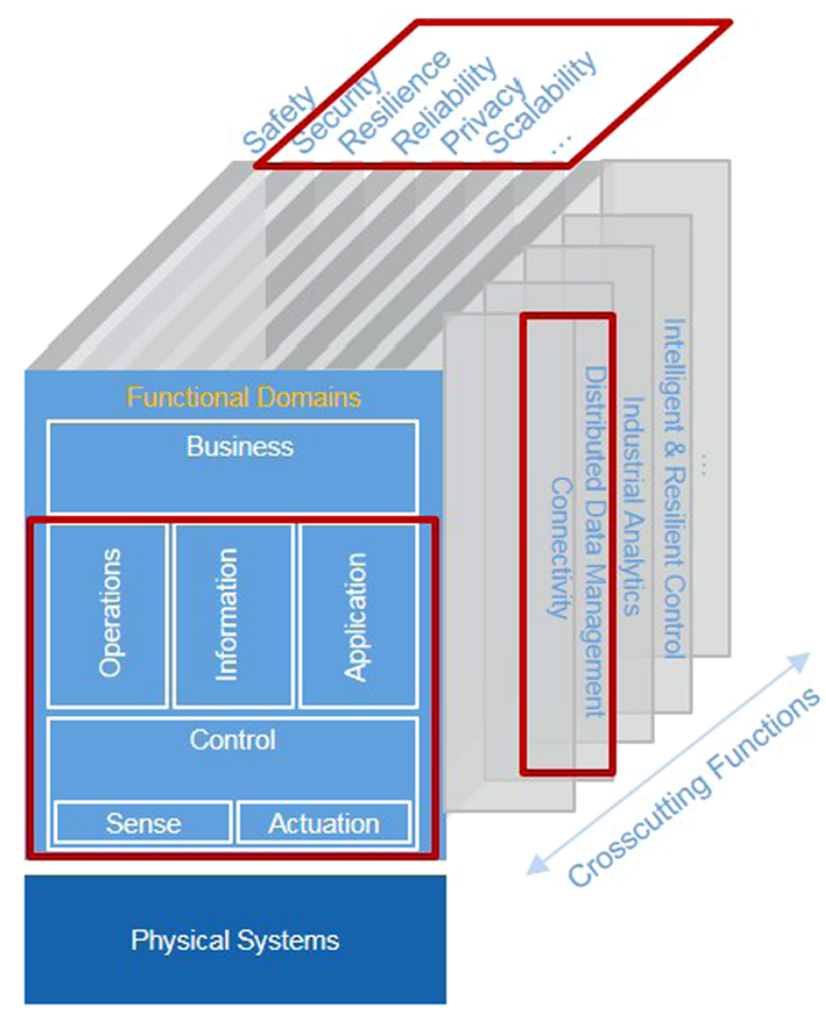

Figure 4: IIRA reference architecture.

pyramid, which structures different layers of responsibility and aggregation from field devices over control hardware to higher-level applications (Manufacturing Execution System (MES), ERP etc.). The Hierarchy Levels enhance the classical pyramid by the categories Product and Connected World, which includes intelligent (communicating) products, as well as interconnecting enterprises and shop floor software technologies to cloud technologies. The industrial networking architecture presented in this paper covers all hierarchy levels, i. e., its offered functionality addresses every layer in the automation pyramid, as well as the connection to superordinate cloud services. 


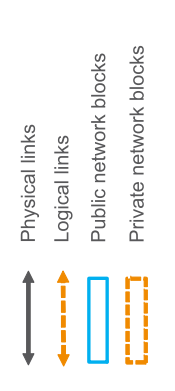


The RAMI4.0 Layers axis divides the solution into six functional levels, of which the communication layer is covered by the architecture presented in this paper. The last dimension of the RAMI4.0 addresses the Life Cycle and Value Stream of products. It divides the product development and the usage process into a type and an instance phase. Whereas the type phase refers mainly to product development including documentation, construction plans, etc., the instance phase refers to the usage phase of the product, in which data is collected during operation. The architecture presented in this paper covers run-time of the $5 \mathrm{G}$ system and hence the instance phase of a product life cycle, which is more challenging from a communication point of view.

\subsection{IIRA}

The IIRA [29] of the Industrial Internet Consortium (IIC) follows ISO/IEC/IEEE 42010:2011 "Systems and Software Engineering-Architecture Description" [30] and contains an IIoT architecture framework, which in turn contains views on stakeholders, concerns and viewpoints as its architecture frame, and views and models as its architecture representation. Considered viewpoints are business, usage, functional and implementation, which help to find stakeholders of an IIoT solution. In general, the IIRA consists of three dimensions: Functional domains, system characteristics and cross-cutting functions (cf. Fig. 4). It considers functional domains, namely control, operations, application, business and information. As highlighted in Fig. 4, our proposed architecture covers all functional domains except the Business domain and all system characteristics except Safety. Our architecture addresses especially the Information functional domain as $5 \mathrm{G}$ allows efficient and high-performance communications. The $5 \mathrm{G}$ system allows information gathering and is part of the cross-cutting functions; however, it is limited to the functions Connectivity and Distributed Data Management contained therein.

\section{Conclusion}

Our use case analysis has shown that a transition from a legacy production factory to the factory of the future is a complex task. It is important to consider the diversity of use cases on the one hand, as well as the requirements imposed by plant owners and managers on the other hand. While $5 \mathrm{G}$ is expected to fulfill the technical requirements, such as data rate and latency, the process requires additional efforts to design and deploy an architecture, which takes into account interoperability of legacy communication technology, operation (business) models of the wireless infrastructure, and maintainability.

In this regard, we proposed a communication network architecture for industrial applications, which combines future $5 \mathrm{G}$ technology and non-cellular network technologies with existing technologies, such as fieldbus, on the shop floor. Through the introduction of IoT Edge Gateways, communication technology aggregation points and by leveraging the benefits of SDN, network slicing and intelligent radio resource management, the architecture is flexible enough to address many different industrial use cases. It is also designed in a way to reduce human intervention and provides the possibility to operate private and/or public cellular networks depending on the factory owner's needs and capabilities. We have also illustrated that our proposed architecture fits well into the existing architectural frameworks and builds upon existing $5 \mathrm{G}$ architectures, which makes it universally suitable framework for future industrial networking.

5Gangs's future work comprises the implementation of system components and the actual numerical evaluation of the overall system performance with regard to the identified requirements.

Acknowledgment: The authors alone are responsible for the content of the paper.

Funding: This work has been supported by the Federal Ministry of Education and Research of the Federal Republic of Germany (Foerderkennzeichen 16KIS0725K, 5Gang).

\section{References}

1. “5G: Industrielles Internet," https://www.bmbf.de/ foerderungen/bekanntmachung-1044.html, accessed 2019-02-13.

2. “5Gang - $5 \mathrm{G}$ angewandt in der Industrie," https:// 5gangprojekt.com/, accessed 2019-02-13.

3. "Minimum requirements related to technical performance for imt 2020 radio interface(s)," https://www.itu.int/md/R15SG05-C-0040/en, accessed 2019-02-13.

4. E. Dutkiewicz, X. Costa-Perez, I. Z. Kovacs and M. Mueck, IEEE Network, vol. 31, no. 6, pp. 6-7, 2017.

5. C. Bockelmann, N. Pratas, H. Nikopour, K. Au, T. Svensson, C. Stefanovic, P. Popovski and A. Dekorsy, "Massive machine-type communications in 5g: Physical and mac-layer solutions," IEEE Communications Magazine, vol. 54, no. 9, pp. 59-65, 2016. 
6. Z. Dawy, W. Saad, A. Ghosh, J. G. Andrews and E. Yaacoub, "Toward massive machine type cellular communications," IEEE Wireless Communications, vol. 24, no. 1, pp. 120-128, 2017.

7. S.-Y. Lien, S.-L. Shieh, Y. Huang, B. Su, Y.-L. Hsu and H.-Y. Wei, "5g new radio: Waveform, frame structure, multiple access, and initial access," IEEE communications magazine, vol. 55, no. 6, pp. 64-71, 2017.

8. "Architecture enhancements for control and user plane separation of epc nodes; stage 2 (release 15)," https://portal.3gpp.org/desktopmodules/Specifications/ SpecificationDetails.aspx?specificationld=3077, accessed 2019-02-13.

9. Software-defined networking: The new norm for networks, Open Networking Foundation, 2012.

10. "Architecture enhancements for dedicated core networks; stage 2 (release 13)," https://portal.3gpp.org/desktopmodules/ Specifications/SpecificationDetails.aspx?specification $I d=868$, accessed 2019-02-13.

11. “3gpp tr 23.711, enhancements of dedicated core networks selection mechanism," http://www.3gpp.org/DynaReport/ 23711.htm, accessed 2019-02-13.

12. H. Wu, G. T. Nguyen, A. K. Chorppath and F. H. Fitzek, "Network slicing for conditional monitoring in the industrial internet of things," IEEE Software Defined Networks, IEEE Softwarization, Jan 2018.

13. I. F. Akyildiz and X. Wang, "A survey on wireless mesh networks," IEEE Communications magazine, vol. 43, no. 9 , pp.S23-S30, 2005.

14. $5 G$ white paper, NGMN Alliance, 2015.

15. S. Redana, A. Kaloxylos, A. Galis, P. Rost and V. Jungnickel, "View on $5 \mathrm{~g}$ architecture," White paper of the 5G-PPP architecture WG (2016, July), 2016.

16. J. Kim, D. Kim and S. Choi, "3GPP SA2 architecture and functions for $5 \mathrm{G}$ mobile communication system," vol. 3, no.1, pp.1-8, http://www.sciencedirect.com/science/article/pii/ S240595951730019X, accessed 2019-02-13.

17. “5G-ACIA Website," https://www.5g-acia.org/, accessed 2019-01-09.

18. White Paper - $5 G$ for Connected Industries and Automation Second Edition, 5G-ACIA, 2018.

19. C. Bockelmann, A. Dekorsy, A. Gnad, L. Rauchhaupt et al., "Hiflecs: Innovative technologies for low-latency wireless closed-loop industrial automation systems," in 22. VDE-ITG-Fachtagung Mobilkommunikation, Osnabrück, Germany, May 2017.

20. B. Holfeld, D. Wieruch, T. Wirth, L. Thiele, S. A. Ashraf, J. Huschke, I. Aktas and J. Ansari, "Wireless communication for factory automation: an opportunity for lte and $5 \mathrm{~g}$ systems," IEEE Communications Magazine, vol. 54, no. 6, pp. 36-43, 2016.

21. L. Rauchhaupt, D. Schulze, A. Gnad and M. Krätzig, "Requirement profiles in zdki," Accompanying Research Reliable wireless communication in industry (BZKI), Tech. Rep., oct 2016.

22. I. Aktas, A. Bentkus, F. Bonanati et al., "Wireless technologies for industrie 4.0," Frankfurt am Main, 2017.

23. 3GPP, "Study on Communication for Automation in Vertical domains (CAV)," https://portal.3gpp.org/desktopmodules/ Specifications/SpecificationDetails.aspx?specificationld= 3187, accessed 2019-02-13.
24. "System architecture for the $5 \mathrm{~g}$ system (5gs)," https://portal.3gpp.org/desktopmodules/Specifications/ SpecificationDetails.aspx?specification Id=3144, accessed 2019-02-13.

25. B. Pfaff, J. Pettit, T. Koponen, E. J. Jackson, A. Zhou, J. Rajahalme, J. Gross, A. Wang, J. Stringer, P. Shelar et al., "The design and implementation of open vswitch," in NSDI, vol.15, 2015, pp. 117-130.

26. S. Jeschke, C. Brecher, T. Meisen, D. Özdemir and T. Eschert, "Industrial internet of things and cyber manufacturing systems," in Industrial Internet of Things, Springer, 2017, pp. 3-19.

27. P. Adolphs, "Rami 4.0 - an architectural model for industrie 4.0," Platform Industrie, vol. 4, 2015.

28. M. Hankel, "Industrie 4.0: The reference architectural model industrie 4.0 (rami 4.0)," Frankfurt am Main, Germany: ZVEI-German Electrical and Electronic Manufacturers' Association, 2015.

29. S.-W. Lin, B. Miller, J. Durand, R. Joshi, P. Didier, A. Chigani, R. Torenbeek, D. Duggal, R. Martin, G. Bleakley et al., "Industrial internet reference architecture," Industrial Internet Consortium (IIC), Tech. Rep, 2015.

30. Systems and Software Engineering, Architecture Description, ISO/IEC/IEEE International Standard ISO/IEC/IEEE 42 010:2011.

\section{Bionotes}

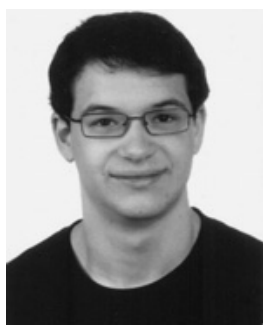

\section{Michael Karrenbauer}

Chair for Wireless Communications and Navigation, University of Kaiserslautern, Kaiserslautern, Germany karrenbauer@eit.uni-kl.de

Michael Karrenbauer received the Dipl.-Ing. degree (M. Sc.) from the University of Kaiserslautern, Germany, in 2013, where he is currently pursuing the Ph. D. degree with the Institute of Wireless Communication and Navigation. He is currently a Research Staff Member with the Institute of Wireless Communication and Navigation where he is working within the German research project $5 \mathrm{Gang}$. His research interests are in the area of reliable communication systems for industrial environments.

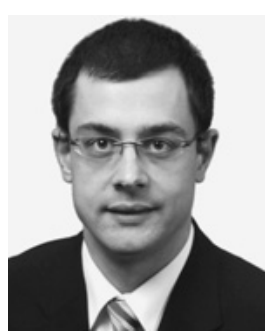

\section{Stephan Ludwig}

Robert Bosch GmbH, Renningen, Germany stephan.ludwig2@de.bosch.com

Stephan Ludwig received his Dipl.-Ing. degree in electrical engineering from the University of Ulm, Germany, in 2007. From thereon, 
he worked as a research assistant on communications engineering at the University of the Federal Armed Forces in Munich, Germany. Since 2014 he has been research engineer with the corporate research sector of Robert Bosch in Renningen, Germany, working on signal processing and on bringing $5 \mathrm{G}$ to factory automation.

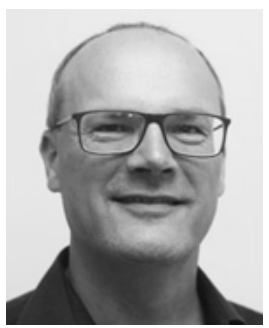

\section{Henning Buhr}

Ericsson $\mathrm{GmbH}$, Herzogenrath, Germany henning.buhr@ericsson.com

Henning Buhr received his diploma in telecommunications engineering from the university of applied science of the Deutsche Telecom in Berlin, Germany, in 1996. He joined Ericsson and worked in different positions within development, project management and product management in Germany and Sweden. Currently he holds a position as a Cloud Technology Specialist in the Cloud Systems and Technology department in Aachen and leads the $5 \mathrm{Gang}$ research project. His actual work areas focus on $5 \mathrm{G}$ technologies and Distributed Cloud.

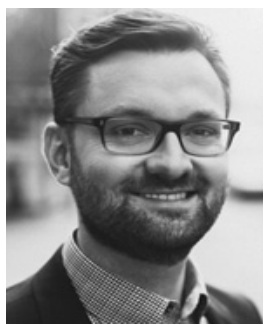

\section{Henrik Klessig}

Robert Bosch GmbH, Renningen, Germany henrik.klessig@de.bosch.com

Henrik Klessig received his M. Sc. (Dipl.-Ing.) in Information Systems Engineering and his Ph. D. (Dr.-Ing.) in Electrical Engineering from Technical University Dresden, Germany, in 2012 and 2016, respectively. He was a research intern at Bell Labs, Stuttgart, Germany, in 2010 and a research associate at the Vodafone Chair, TU Dresden between 2012 and 2016, where he has been working on queuing and traffic theory and its application to self-organizing networks. He was a recipient of the FITweltweit postdoc stipend from the German Academic Exchange Service and has been working on edge computing service continuity and aerial wireless networks at the International Computer Science Institute. He was also a visiting researcher at UC Berkeley in 2018. Since December 2018, he has been with Robert Bosch GmbH in Renningen, Germany.

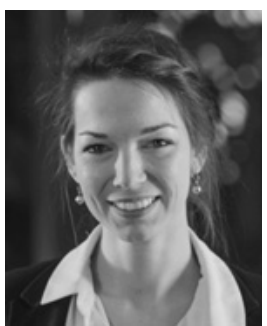

Anne Bernardy

FIR e. V. at RWTH Aachen, Aachen, Germany anne.bernardy@fir.rwth-aachen.de

Anne Bernardy was born in Gerolstein in 1989. She studied mechanical engineering focusing construction and design for medical assets and microsensors at the RWTH Aachen University. Meanwhile, she gained experience in the development of optical systems and their application because of her work as a research assistant at the Fraunhofer IPT in Aachen. After receiving the master's degree in 2014, she began to work as a researcher at the FIR Institute of Industrial Management of the RWTH Aachen University. Her main topic is the matching between industrial use cases with the Internetof-Things and suitable, innovative information and communication technologies.

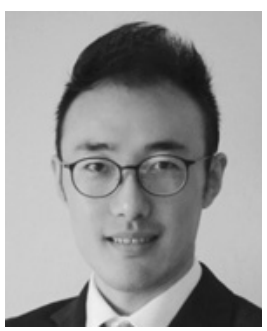

\section{Huanzhuo Wu}

Deutsche Telekom Chair of Communication Networks, Technische Universität Dresden, Dresden, Germany huanzhuo.wu@tu-dresden.de

Huanzhuo Wu received his M. Sc. degree in Computer Science at Technical University Dresden, Germany, in 2016, he is currently pursuing his Ph. D. degree in Electrical Engineering at the Technical University Dresden, Germany. Now he is a research fellow at Deutsche Telekom Chair of Communication Networks. He is currently working within the German research project $5 \mathrm{Gang}$.

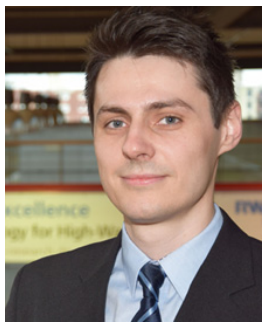

Christoph Pallasch

Laboratory for Machine Tools and Production Engineering, RWTH Aachen, Aachen, Germany c.pallasch@wzl.rwth-aachen.de

Christoph Pallasch received his Master of Science degree in Computer Science from the RWTH Aachen University, Germany, in 2013. He is employed at the Laboratory for Machine Tools and Production Engineering (WZL) since 2015 and works towards his Ph. D. degree, researching in Wireless Communication technologies, Plug-andProduce as well as industrial automation and control. 


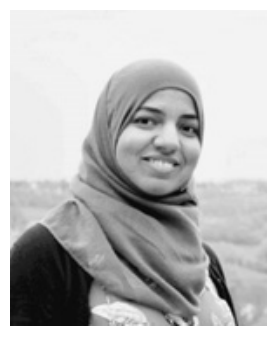

\section{Amina Fellan}

Chair for Wireless Communications and Navigation, University of Kaiserslautern, Kaiserslautern, Germany

fellan@eit.uni-kl.de
Amina Fellan received her M. Sc. in Communication Engineering from RWTH Aachen University, Germany, in 2015. She is currently working as a research assistant at the University of Kaiserslautern. Her research interests include software-defined-radios and dynamic resource allocation in $5 \mathrm{G}$ networks.

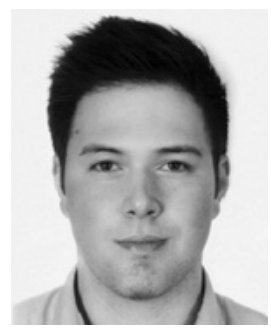

\section{Nicolai Hoffmann}

Laboratory for Machine Tools and Production Engineering, RWTH Aachen, Aachen, Germany n.hoffmann@wzl.rwth-aachen.de

Nicolai Hoffmann received his Master of Science degree in Production Engineering from the RWTH Aachen University, Germany, in 2017. He is currently employed at the Laboratory for Machine Tools and Production Engineering (WZL) and works towards his Ph. D. degree, researching in Robotics, Additive Manufacturing, Industrial Internet-of-Things and Wireless Communication technologies for industrial automation.

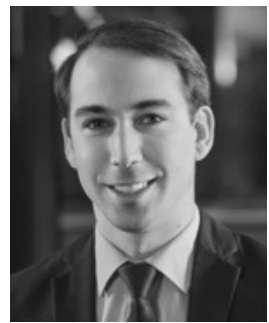

\section{Vasco Seelmann}

FIR e. V. at RWTH Aachen, Aachen, Germany vasco.seelmann@fir.rwth-aachen.de

Vasco Seelmann was born in Erlangen, Germany, in 1990. He received the B. Sc. degree and the M. Sc. degree from RWTH Aachen University, both in business administration and mechanical engineering, where he focused on aerospace engineering. He is pursuing the Ph. D. degree with the FIR Institute for Industrial Management at RWTH Aachen University. His current research focusses on identifying and developing innovative applications of information and communication technology in industrial environments.

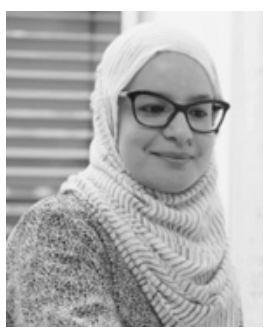

\section{Maroua Taghouti}

Deutsche Telekom Chair of Communication Networks, Technische Universität Dresden, Dresden, Germany

maroua.taghouti@tu-dresden.de

Maroua Taghouti received her Engineering degree in Telecommunications in 2012 from the National Engineering School of Tunis, followed by a master of research in Communications Systems. Before that she studied Mathematics and Physics for two years in the Institute of Preparatory Studies for Engineering of Tunis. Currently, she is a Ph. D. candidate at Tunisia Polytechnic School, and working simultaneously as a research fellow at the Deutsche Telekom Chair of Communications Networks in the Technical University of Dresden. Her research interests focus on coding theory, mainly Network Coding, and Compressed Sensing for communications and networking. She served as a reviewer for different conferences and journals including IEEE Communications Letters and IEEE Communications Surveys and Tutorials.

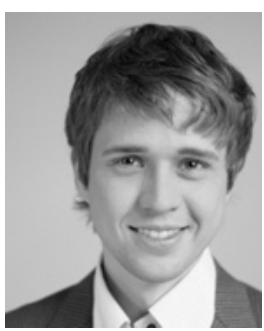

\section{Simon Wunderlich}

Deutsche Telekom Chair of Communication Networks, Technische Universität Dresden, Dresden, Germany simon.wunderlich@tu-dresden.de

Simon Wunderlich is currently pursuing his Ph. D. degree in Electrical Engineering at the Technical University Dresden, Germany. He received his Dipl.-Inf. degree in Computer Science at Chemnitz Technical University, Germany, in 2009. He is co-author of the Wi-Fi Mesh software B.A.T.M.A.N. Advanced.

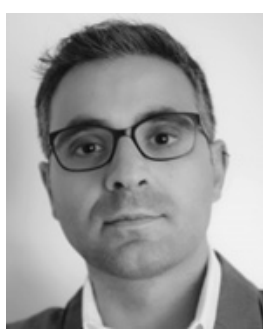

\section{Pedro Tercero Lozano}

Ericsson GmbH, Herzogenrath, Germany pedro.tercero.lozano@ericsson.com

Pedro Tercero Lozano received his BSc in Telecommunications Engineering from Polytechnics University of Alcala de Henares, Spain, in 2005 and his Executive Program in IT management from Solvay Business School, Belgium, in 2013. Since 2007 he has worked as end-to-end mobile networks system architect. He joined Ericsson Eurolab Germany GmbH in 2012 where he has held several technical and lead positions developing solutions for different wireless technologies (including Wi-fi, LTE and 5G) and contributing in filing several patents applications. Currently, he supports the German research project $5 \mathrm{Gang}$ as overall test network responsible. 


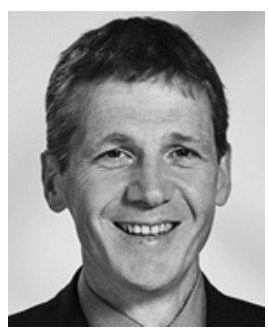

\section{Andreas Hoell}

SICK AG, Waldkirch, Germany

andreas.hoell@sick.de
Andreas Hoell has been with SICK AG since 2001. He started his career with safety network integration applications. Since 2007, he has worked as Technical Industry Manager in factory automation with an emphasis on automotive industry. His additional interest is in research projects for Industry 4.0 and automated production networks.

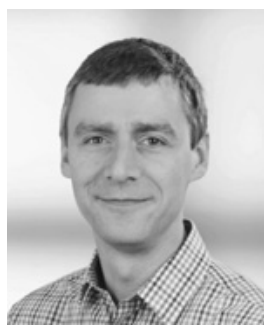

\section{Christian Stimming SICK AG, Waldkirch, Germany christian.stimming@sick.de}

Christian Stimming received his Dipl.-Ing. degree in electrical engineering from the Hamburg University of Technology, Germany, in 2001, and his Ph. D. in 2007. From thereon, he has been a research engineer with SICK AG the corporate research department in Hamburg, Germany, working on signal processing algorithms and future wireless services in factory automation.

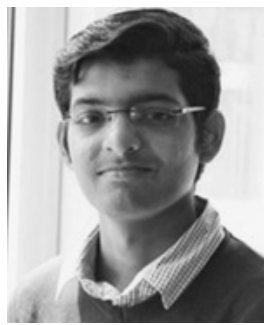

\section{Dhruvin Patel}

Ericsson GmbH, Herzogenrath, Germany dhruvin.patel@ericsson.com

Dhruvin Patel received his Master of Science degree (M. Sc.) in Computer and System Engineering from the Technical University of IImenau, Germany in 2016. He is currently employed at Research department in Ericsson $\mathrm{GmbH}$, Aachen as a Researcher. Scope of his research work includes developing new features to enable next generation mobile networks for industrial automation.

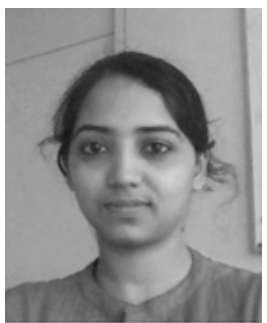

Savita Seetaraman

Ericsson GmbH, Herzogenrath, Germany savita.seetaraman@ericsson.com

Savita Seetaraman received her dual masters from EIT Digital Master School in Computer Science and Innovation and Entrepreneurship in 2017. She is currently working as a Cloud Developer in Ericsson GmbH Aachen. Her areas of work include Distributed Cloud Solution Development and Cloud Application Orchestration.

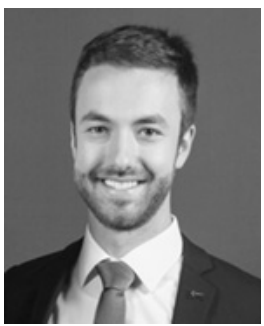

\section{Serjoscha Bender} Ericsson $\mathrm{GmbH}$, Herzogenrath, Germany serjoscha.bender@ericsson.com

Serjoscha Bender is currently pursuing his master's degree in Computer Science at RWTH Aachen University where he received his B. Sc. in 2018. As a working student at Ericsson GmbH in Aachen he is supporting the research on the use of mobile networks for industry communication and the development of distributed cloud solutions.

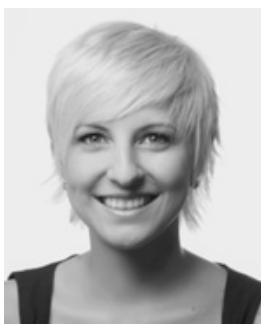

\section{Elena Eberhardt \\ Schildknecht AG, Murr, Germany \\ elena.eberhardt@schildknecht.ag}

Elena Eberhardt graduated from University of Applied Science in Mosbach in 2010 as an industrial engineer (B. Eng.) and received a MBA degree from Mannheim Business School in 2015. She worked in Sales, Marketing and Business Development in the industry sector in Germany and Turkey. Since 2015 she has worked for Schildknecht AG as a Marketing and Business Development Manager to implement radio and communication solutions. She supports the project $5 \mathrm{Gang}$ with business models to implement $5 \mathrm{G}$ for industrial applications. 


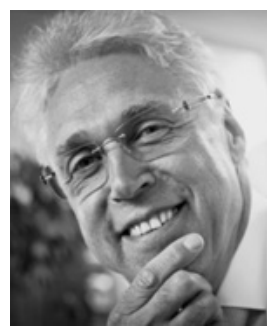

\author{
Thomas Schildknecht \\ Schildknecht AG, Murr, Germany \\ thomas.schildknecht@schildknecht.ag
}

Thomas Schildknecht received his Dipl. Ing. degree 1980 at the University of Dieburg/Darmstadt. In 1981 he founded his own engineering company for industrial hard and software development. Starting with the first project in the automation industry for $\mathrm{HMI}$, the company began in 1993 focusing on own products for wireless solution in industrial applications. 2009 all business activities was brought in the Schildknecht AG, a share based, not stock listed, capital company. Thomas is shareholder, CEO and CTO. In the VDE/GMA committee 5.21 he has been since 2000 member and coauthor for VDI/VDE 2185 Guideline part 2 and 4 for radio based industrial automation. 2007 a patent was granted for a procedure of tunneling fieldbuses via a radio link.

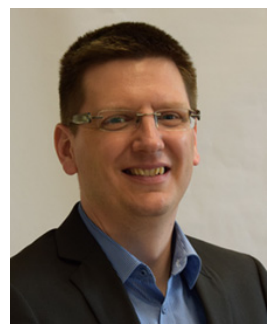

\section{Werner Herfs}

Laboratory for Machine Tools and Production Engineering, RWTH Aachen, Aachen, Germany

w.herfs@wzl.rwth-aachen.de

Werner Herfs Academic Chairman and Executive Chief Engineer of the Chair of Machine Tools at Laboratory for Machine Tools and Production Engineering (WZL) at RWTH Aachen University. He was a research associate between 2003 and 2006. Until 2010, he has been the head of the department Automation and Control related to the Chair of Machine Tools.

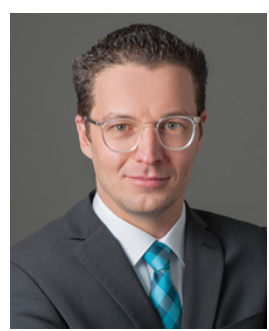

\section{Simon Storms}

Laboratory for Machine Tools and Production Engineering, RWTH Aachen, Aachen, Germany s.storms@wzl.rwth-aachen.de

Simon Storms is head of the department Automation and Control related to the Chair of Machine Tools at Laboratory for Machine Tools and Production Engineering (WZL) at RWTH Aachen University. He was a research associate between 2014 and 2018, after receiving his Master degree in Automation Engineering at the RWTH Aachen University. Until 2018, he has been team leader for automation technologies in the department Automation and Control. His research priorities are robotic applications, industrial communication technologies, Cloud-Computing, flexible process controls, model based engineering and human-machine interaction.

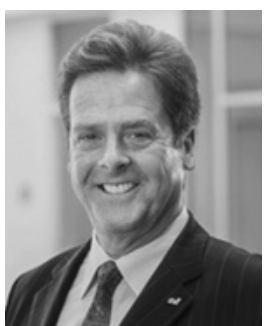

\section{Volker Stich}

FIR e. V. at RWTH Aachen, Aachen, Germany

volker.stich@fir.rwth-aachen.de

Volker Stich studied at the RWTH Aachen University receiving the degree as a "Dipl.-Ing." and continued his academic career by receiving the Ph. D. for his research about the industrial management of logistics. Thereafter, he worked for an automobile OEM as a leader of the manufacturing logistics. Since January 1997, he has been the managing director of the FIR e. V. at the RWTH Aachen University. Therefore, he got an extraordinary degree as a Professor. Additionally, he leads the Cluster Smart Logistik at the RWTH Aachen Campus and coordinates the collaboration between research institutes, federations and companies. As a professor, he manages several courses at the university and participate in federations and associations.

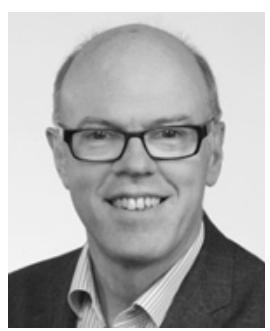

\author{
Norbert Niebert \\ Ericsson GmbH, Herzogenrath, Germany \\ norbert.niebert@ericsson.com
}

Norbert Niebert received the Diploma and Ph. D. degrees in electrical engineering from the Aachen University of Technology RWTH, Germany, in 1985 and 1991, respectively. In 1991 he joined Ericsson where he held various positions in Research and also served as project and technical manager to a number of EU co-financed projects like Mobilise, Ambient Networks and 4WARD. Since 2016 he has been heading the Cloud Systems and Technology work in Aachen and has been an initiator of the $5 \mathrm{Gang}$ project. His current research interests are in the areas of distributed cloud, $5 \mathrm{G}$ applications and innovations in smart manufacturing.

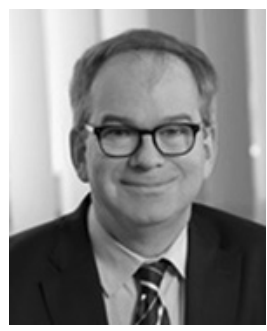

\section{Hans D. Schotten}

Chair for Wireless Communications and Navigation, University of Kaiserslautern, Kaiserslautern, Germany schotten@eit.uni-kl.de

Hans D. Schotten received the Diploma and Ph. D. degrees in electrical engineering from the Aachen University of Technology RWTH, Germany, in 1990 and 1997, respectively. He held positions, such as senior researcher, project manager, and head of research groups at the Aachen University of Technology, Ericsson Corporate Research, and Qualcomm Corporate Research and Development, respectively. 
At Qualcomm, he was the Director for Technical Standards and a Research Coordinator for Qualcomm's participation in national and European research programs. He was a Company Representative with WWRF, EICTA, Bmco forum, and European Technology Platforms. Since 2007, he has been a Full Professor and the Head of the Institute of Wireless Communication and Navigation, University of Kaiserslautern. Since 2012, he has additionally been a Scientific Director with the German Research Center for Artificial Intelligence heading the Intelligent Networks Department.

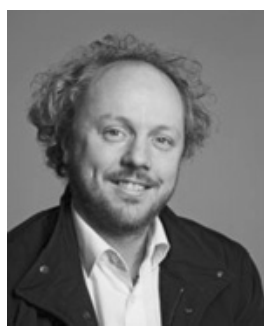

Frank H. P. Fitzek

Deutsche Telekom Chair of Communication Networks, Technische Universität Dresden, Dresden, Germany

frank.fitzek@tu-dresden.de

Frank Fitzek is a Professor and head of the Deutsche Telekom Chair of Communication Networks at the Technical University Dresden, Germany, coordinating the $5 \mathrm{G}$ Lab Germany. He received his diploma (Dipl.-Ing.) degree in electrical engineering from the University of Technology - Rheinisch-Westfälische Technische Hochschule (RWTH) - Aachen, Germany, in 1997 and his Ph. D. (Dr.-Ing.) in Electrical Engineering from the Technical University Berlin, Germany in 2002 and became Adjunct Professor at the University of Ferrara, Italy in the same year. In 2003 he joined Aalborg University as Associate Professor and later became Professor. Dr. Fitzek co-founded several start-up companies starting with acticom $\mathrm{GmbH}$ in Berlin in 1999. He was selected to receive the NOKIA Champion Award several times in a row from 2007 to 2011. In 2008 he was awarded the Nokia Achievement Award for his work on cooperative networks. In 2011 he received the SAPERE AUDE research grant from the Danish government and in 2012 he received the Vodafone Innovation prize. In 2015 he was awarded the honorary degree "Doctor Honoris Causa" from Budapest University of Technology and Economy (BUTE). His current research interests are in the areas of wireless and mobile $5 \mathrm{G}$ communication networks, mobile phone programming, network coding, cross layer as well as energy efficient protocol design and cooperative networking. 